\title{
Comparative microbial community analysis of fur seals and salmon aquaculture in Tasmania
}

\author{
Erin D'Agnese ${ }^{1}$, Ryan McLaughlin² ${ }^{2}$ Mary-Anne Lea ${ }^{3}$, Esteban Soto ${ }^{1}$, Woutrina Smith ${ }^{1}$, \\ and John Bowman ${ }^{3}$ \\ ${ }^{1}$ University of California Davis \\ ${ }^{2}$ Practical Informatics \\ ${ }^{3}$ University of Tasmania
}

October 12,2020

\begin{abstract}
In Tasmania, Australia, the farmed Atlantic salmon (Salmo salmar L) industry utilizes marine leases to cultivate stock and faces opportunistic predation pressure from wild Australian fur seals (Arctocephalus pusillus doriferus). Microbial connectivity between aquaculture and wild pinnipeds was explored during this research, to find potential indicators for microbial sharing, identify potential pathogens at this interface, and elucidate potential effects of interactions on the microbiota of wild seals. High-throughput sequencing of the V1-V3 region of the 16S rRNA genes found in the gut microbial communities of 221 fur seals was performed; 41 males caught at fish farms, 50 fresh adult scats from haul-outs near farms, 24 necropsied seals, and controls from Bass strait rookeries including: 56 adult scats and 50 pup swabs. QIIME2 and R software were used for analysis of fur seal microbiomes, and to compare seal microbiomes to previously collected salmon microbiomes. Foraging at farms appears to shift biodiversity in fur seal microbiota; seals foraging at or near farms having greater phylogenetic diversity in their gut microbial communities compared to control seals. Taxonomic analysis showed a greater divergence in Proteobacteria representatives in male seals captured at farms compared to all other groups and identified indicator OTUs which could be used as minimally invasive indicators for interactions at this interface. These indicator OTUs included members of the following taxa: Clostridium sensu stricto I, Psychrobacter, Xanthomonadaceae, Suttonella, Pelomonas, an unclassified Weeksellaceae genus, Edwardsiella hoshinae, Pleisomonas shigelloides, and Cetobacterium ceti. Several potential pathogens were identified to monitor for human and animal health.
\end{abstract}

\section{Comparative microbial community analysis of fur seals and salmon aquaculture in Tasmania}

Erin D'Agnese ${ }^{1,4,5}$, Ryan J. McLaughlin², Mary-Anne Lea ${ }^{3}$, Esteban Soto ${ }^{1}$, Woutrina Smith ${ }^{1}$, John P. Bowman $^{4}$

${ }^{1}$ University of California, Davis, School of Veterinary Medicine, 1089 Veterinary Medicine Dr, Davis, CA 95616, USA

${ }^{2}$ Practical Informatics, 1721 Electric Ave, Bellingham, WA, 98229, USA

${ }^{3}$ University of Tasmania, Institute for Marine and Antarctic Studies, Battery Point, Tasmania, Australia, 7005

${ }^{4}$ University of Tasmania, Tasmania Institute of Agriculture, Life Sciences Building, Sandy Bay, Tasmania, Australia 7005

${ }^{5}$ Corresponding author: erdagnese@ucdavis.edu

\section{Abstract}


In Tasmania, Australia, the farmed Atlantic salmon (Salmo salmarL) industry utilizes marine leases to cultivate stock and faces opportunistic predation pressure from wild Australian fur seals (Arctocephalus pusillus doriferus ). Microbial connectivity between aquaculture and wild pinnipeds was explored during this research, to find potential indicators for microbial sharing, identify potential pathogens at this interface, and elucidate potential effects of interactions on the microbiota of wild seals. High-throughput sequencing of the V1-V3 region of the $16 \mathrm{~S}$ rRNA genes found in the gut microbial communities of 221 fur seals was performed; 41 males caught at fish farms, 50 fresh adult scats from haul-outs near farms, 24 necropsied seals, and controls from Bass strait rookeries including: 56 adult scats and 50 pup swabs. QIIME2 and $\mathrm{R}$ software were used for analysis of fur seal microbiomes, and to compare seal microbiomes to previously collected salmon microbiomes. Foraging at farms appears to shift biodiversity in fur seal microbiota; seals foraging at or near farms having greater phylogenetic diversity in their gut microbial communities compared to control seals. Taxonomic analysis showed a greater divergence in Proteobacteria representatives in male seals captured at farms compared to all other groups and identified indicator OTUs which could be used as minimally invasive indicators for interactions at this interface. These indicator OTUs included members of the following taxa: Clostridium sensu stricto I, Psychrobacter, Xanthomonadaceae, Suttonella, Pelomonas, an unclassified Weeksellaceae genus, Edwardsiella hoshinae, Pleisomonas shigelloides, and Cetobacterium ceti . Several potential pathogens were identified to monitor for human and animal health.

Keywords: Australian fur seal, microbiome, aquaculture, Tasmania, One Health, microbial source tracking, Atlantic Salmon

\section{Introduction}

Reliance on the marine and coastal regions for aquaculture has increased in recent decades (Msangi \& Batka, 2015; Stevens, Newton et al. , 2018). In the marine system, humans have historically been directly connected to the wild system in terms of fisheries of wild fish stocks and direct interaction with wild animals through recreational activities, fisheries competition for fish stocks, and aquaculture (Gulland, 1987; Kemper et al., 2003; Bowen \& Lidgard, 2013). However, the indirect connections are often more insidious and easily overlooked. The interconnectedness of the wild system to humans, both directly and indirectly, has become more evident as aquaculture management in nearshore systems is improved upon. In Tasmania, an island state of Australia, the farmed Atlantic salmon (Salmo salar L.) industry produced 51,298 tonnes of salmon in 2016-2017, and production has continued to increase annually. The industry in Tasmania alone produces around $\$ 739$ million of profit and has become the single most important aquaculture commodity in Australia (Mobsby, 2018; Pattersonet al. , 2018). Of the salmon produced, 5,037 tonnes were exported to other countries in 2016-2017. This industry continues to grow in the region, meanwhile, the Australian fur seal (Arctocephalus pusillus doriferus) and Long-nosed fur seal (Arctocephalus forsteri) populations have been rebounding from seal hunting, when their populations were reduced to near extinction (Kirkwood et al. , 2010). While growth has slowed, populations remain reduced post-seal hunting, and their continued recovery remains tenuous as they face the modern human impacts on their ecosystem. The interactions between fur seals and aquaculture leads directly to increased risk for the safety of fish, human and marine mammals in many similar systems around the world (Kemper et al. , 2003; Nash et al. , 2000; Wiirsig \& Gailey, 2002). Often the Australian fur seals in Tasmania predate on farmed salmon, leading to reduction in salmon stock, risk to farm workers, and occasionally these interactions can lead to the injury and mortality of the seals (Pemberton \& Shaughnessy, 1993; Robinsonet al. , 2008). Direct impacts are currently being mitigated by regulation and by the industry improving pen infrastructure, however the extent of indirect effects and risks are largely unknown. At this interface, little is known about the indirect effects, such as the microbial connections between aquaculture and wildlife.

Microbial source tracking (MST) systems have been employed to follow pathogens and sources of pollution into the aquatic and marine ecosystems. Historically MST systems have relied on faecal coliforms and indicator bacteria, such as E. coli, to follow terrestrial contamination through aquatic and marine systems, as well as, determine water quality/safety (Geldreich \& Litsky, 1976; Scott et al ., 2002). However, these techniques have many limitations in their ability to discern point sources, and the use of genetic markers and genetic 
libraries have been adopted as more precise in measuring point sources as molecular methodologies continue to advance (Scott et al. , 2002). Often these techniques are labor-intensive and have their own limitations in fully understanding the interplay between multiple sources and identifying all sources of contamination (Ahmed, 2007; Griffith, Weisberg, \& McGee, 2003). With advances in technology, and analysis software development, the ability to track multiple taxa in microbial communities through systems has become possible (Bourneet al. , 2013; Kembel et al. , 2012; Knights et al. , 2011). Metacommunity analysis utilizes community diversity of species in communities, and community structure changes, to elucidate the extent of interactions in communities, including the microbial communities found in nature (Holyoak, Leibold et al. , 2005; Miller et al. , 2018). Comparing full microbial communities between regions and trophic levels as a way of analysing bacterial movements, is an up and coming field of research (Unno et al. , 2010; 2011).

Microbiomes are the communities of microbial organisms living in distinct environments such as in or on another organism such as in the guts or on the skin (Barko et al. , 2018; Grice \& Segre, 2011). Microbes, such as bacteria and fungi, thrive in a wide variety of environments, depending on their specific growth requirements and characteristics. Many are shared through trophic systems, from prey to predator, and up the food chain (Christian et al. , 2015; Xu \& Knight, 2015). Some microbes are regionally more or less abundant, and their presence in a host can indicate whether that animal has spent time in that region (Alfano et al. , 2015; Martinez-Guryn et al. , 2019). Studying microbiomes has the capability to elucidate what an animal is eating, what environments it is encountering, and what pathogens it has been exposed to, all in one detailed analytical study.

Differences in nutrition, age and regionality are known to affect microbiome structure and diversity in many species including pinnipeds (Ley et al. , 2008; Hills et al. , 2019; Pacheco-Sandovalet al. , 2019). As an animal ages, the microbiota shifts with increasing microbial input from the environment, and their changing diet, these changes throughout their life alter the makeup of bacterial communities' animals interact with. In adult animals the microbiome is more established and bigger changes in the environment and diet are needed to greatly shift the microbiome. However, it is known that when a substantial shift in diet is made and then maintained the microbial community can change drastically. As salmonids are not a natural prey item for wildlife in Tasmania, and the microbial communities found in and on salmon are now entering predators' gastrointestinal tract, it is likely that foraging on farmed salmon is going to lead to a change in overall microbiome structure. The microbes in farmed salmon have the potential to be transferred to the animals and people which eat them.

This study aimed to elucidate the microbial community shifts in seals microbiomes associated with foraging on farmed salmon. The microbiomes of Australian fur seals and stranded Long-noses/New Zealand fur seals found in Tasmania were compared across age class, location of sampling, and their proximity to salmon farms to determine diversity differences, and detect the operational taxonomic units (OTU)s, which drive the community diversity changes found. The microbiomes of seals were compared to a representative sampling of farmed salmon microbiomes to determine if seals foraging on farmed salmon have more similar microbial communities and OTUs to the salmon compared to other seals in Tasmania. Microbes shared between farmed salmon and seals were identified through this comparative analysis.

\section{Methods}

\section{Sample collection}

Male fur seals $(n=41)$ were trapped at three salmon farm leases in the southern region of the state by the permitted farm managers following the protocol set by the state government animal ethics committee, then handled. Seals were trapped both inside and outside of salmon pens using baited pens which close after a seal has grabbed the bait at the back of the trap, held overnight or up to 36 hours in pens on land, until sampling occurred under permits from the Department of Primary Industries, Parks, Water and Environment (2017/18: FA 17158 and 2018/19: FA 18114) and all handling of animals occurred under University of Tasmania Animal ethics (UTAS AEC project number: A0016754). At time of collection, seals were sedated with Hypnovel ( $5 \mathrm{mg} / \mathrm{ml}$ Midazolam) (Roche products Pty Limited, Sydney, NSW, Australia) at 
a dose rate of $0.35-0.6 \mathrm{mg} / \mathrm{kg}$ depending on size of animal and state of excitement. Rectal swabs were taken from each male, two were placed in RNAlater like buffer. If the seal defecated while under sedation, feces was collected in addition to swabs. The swabs/feces in RNAlater were frozen at $-20^{\circ} \mathrm{C}$ for later extraction.

Pups were swabbed at Tenth Island in January $2018(\mathrm{n}=50)$. The sample handling and storage were the same as per above for males sampled at the farms. Feces from adults on Tenth Island and Judgement Island in January 2018 were also collected $(n=56)$ in $0.9 \%$ saline and RNAlater as per the swab methods, fecal samples were collected by swabbing the outside of fresh feces to obtain approximately $500-800 \mathrm{mg}$ of feces. Throughout winter 2018 (May-August), whole fecal samples were collected by collaborators, and then 50 random samples of fresh scats from islands in the southeast of Tasmania were subsampled in RNAlater to be included in the data set. Necropsies were performed on fresh dead, and moderately decomposed carcasses of fur seals from around Tasmania during this study, and fecal samples were collected in RNAlater to be included in this study $(\mathrm{n}=24)$.

\section{DNA extraction and sequencing}

Extraction of DNA from fecal samples was performed as per manufacturer protocol using Qiagen DNeasy mini stool kit or the updated version of the kit Qiagen Fast stool kit. After extraction, DNA was stored at $-20^{\circ} \mathrm{C}$ until preparation for sequencing. Samples were tested for nucleic acid content by nanodrop using a Thermo Scientific Nanodrop 8000 before sequencing. Samples were then sent for sequencing of the V1-V3 region of the 16S rRNA gene to Ramaciotti Centre for Genomics, Sydney, NSW, Australia. The sequencing was carried out on the amplified product from implementation of the $27 \mathrm{~F} / 519 \mathrm{R}$ universal primers for the V1$\mathrm{V} 3$ region of the $16 \mathrm{~S}$ rRNA gene in order to compare the previously sequenced farmed salmon microbiomes directly, which also utilized the V1-V3 region gene target, and to increase resolution at the genus and species level in gut microbiome analysis (Allen et al. , 2016; Huse et al. , 2012). The representative farmed Atlantic salmon sequences from leases in southern Tasmania which are included in the analysis here, can be found in National Center for Biotechnology Information (NCBI) Sequence Read Archive with accession number SRP133157. Quality control, library preparation, and normalization was carried out before high-throughput sequencing using the MiSeq platform was performed.

Demographic groups for the seal and salmon combined analysis are as follows: salmon, males sampled at fish farms (FF), seals sampled in the region around salmon farms (InRegAd), which includes necropsied animals and feces collected from haul-outs in the same region of fish farms, control adults (feces and necropsied animals sampled from outside the regions around fish farms), and control juveniles (pups sampled at Tenth Island in Bass Strait). The regions around FF and the sampling sites are shown on the map of Tasmania (figure 1).

\section{PCR and Sanger sequencing of enteric isolates for partial reference database construction}

The swabs and feces from seals which were collected in $0.9 \%$ saline were lightly vortexed to suspend any bacteria on the swab into solution, then the solution was serially diluted in sterile $0.9 \%$ saline to $10^{-1}$ and $10^{-2}$. These dilutions were then plated on brain heart infusion (BHI) agar, marine agar, thiosulfate-citratebile salts-sucrose agar (TCBS), xylose lysine deoxycholate (XLD) and eosin methylene blue agar (EMB) and grown for 48 hours. After 48 hours morphologically distinct colonies from each agar type were lifted for isolation on BHI agar or marine agar dependent on the initial growth media. Isolate DNA was extracted for PCR and identification via Sanger sequencing by aseptically transferring a pure colony into $400 \mu \mathrm{L}$ of sterile double distilled water and heating at 100 for 10 minutes, then centrifuged for $1 \mathrm{~min}$ at $12000 \mathrm{rpm}$ to pellet cell particles. The supernatant was used as the genomic DNA in PCR reactions. The bacterial 16S rRNA V1-V3 region amplicon was amplified using conventional PCR methodologies with MyTaq red mix, 27F and 519R universal primers at $10 \mu \mathrm{M}$, genomic DNA and sterile double distilled water $(10 \mu \mathrm{L}, 1 \mu \mathrm{L}, 1 \mu \mathrm{L}, 11 \mu \mathrm{L}$ respectively with water added to fill reactions to $25 \mu \mathrm{L}$ ) reactions were run on a Biorad thermocycler with the following parameters: 95 for $1 \mathrm{~min}$, then 30 cycles of 95 for $1 \mathrm{~min}, 55$ for $1 \mathrm{~min}$, and 72 for $1 \mathrm{~min}$, to

finish with 72 for $1 \mathrm{~min}$ and held at 10 until taken off the thermocycler. Sanger sequencing was used to sequence the 16S rRNA gene PCR product at Macrogen Inc., Seoul, South Korea. 


\section{Reference database construction and utilization}

To account for the novelty witnessed through preliminary analysis of the taxonomic classifications of the OTUs in seal gut microbiomes, a database was constructed using the isolates cultured from a subset of the same seals used in this comparative microbiome study. The sequences from the isolates cultured and sequenced via Sanger sequencing were clustered at a 94\% threshold clustering at a genus level. The subsequent representative sequences were classified using the SILVA 132 non-redundant SSU rRNA database, in QIIME 2 (ver. 2019.4). This seal specific reference database created through this method was used in tandem with the SILVA 132 SSU rRNA database when assigning taxonomy to the representative sequences of the seal microbiomes and the full seal and salmon microbiome dataset.

The representative sequences from the seal microbiomes only, and the full dataset including salmon and seals, were classified at a threshold of $97 \%$ similarity to both the aforementioned created isolate database (isolate DB) and SILVA reference database (SILVA DB) using the Qiime2 (Bokulich et al., 2018). To assign the most appropriate classification from the two databases, we compared the percent identification (\% ID) produced from both classifications. To do this systematically and uniformly, the difference between the isolate database value and the SILVA database value was calculated for each representative sequence by subtracting the SILVA \% ID from the isolate DB \% ID. As the isolate database was initially classified using the SILVA database at a $94 \%$ similarity threshold, due to the high level of novelty found in the cultured isolates, calculated \%ID differences between the SILVA database and isolate database assignments were handled in the following ways: (1) The representative sequence was assigned the SILVA DB taxonomic classification if the \% ID difference calculated was less than 0 or it was [?] 0 but $>$ the $\%$ ID calculated from the initial isolate DB \% calculated to the SILVA DB; (2) The representative sequence was assigned the taxonomic classification from the created isolate DB if the \% ID difference was [?]10\%, thus ensuring a much higher similarity to the isolate sequence; (3) the lowest common ancestor shared between the isolate DB and the SILVA DB classifications was assigned to the representative sequence if the difference was $<10 \%$ to avoid misclassification at a higher taxonomic level. Taxonomic tables were constructed from these assignments and were used in downstream analysis of taxonomic differences for both the seals and salmon datasets.

\section{Microbiome Analysis}

All analysis of bacterial 16S rRNA gene amplicon sequence data was carried out using QIIME 2 (ver. 2019.4) and the packages 'tidyverse', 'qiime2R', 'vegan', 'phyloseq', and 'indicspecies' in RStudio employing R v.3.6.1 (Bolyen et al. , 2018; Hall \& Beiko, 2018). Forward and reverse reads for each sample were denoised, edited for quality, and joined using DADA2 (ver 1.14) (Callahan et al. , 2016). Clustering of similar, joined sequences was done using VSEARCH (ver. 2.7.1) and grouped into the most abundant OTUs with a 97\% identity similarity (Rognes et al., 2016). Alpha and beta diversity was calculated for within sample and between group diversity. First, seal samples were analysed for diversity metrics and then diversity differences were calculated with salmon data included to compare seal groups to the salmon microbiomes. The alpha and beta diversity metrics were calculated with a sampling depth of 1000 sequences per sample. The alpha diversity metrics calculated using QIIME 2 to determine within group richness and evenness included Shannon's diversity index, Simpson's diversity index, Observed OTUs, Faith phylogenetic diversity for community richness, and Pielou's Evenness. The beta diversity metrics computed to determine between group diversity dissimilarity included; principal component analysis (PCA) based on Bray-Curtis distance, unweighted and weighted UniFrac distance, PERMANOVA of UniFrac Unweighted distances, and principal coordinates analysis (PCoA) in QIIME2 and visualized in R using 'ggplot2', 'phyloseq' and 'vegan' packages, multidimensional scaling (MDS) was performed and plotted in $\mathrm{R}$ with the same packages (McMurdie \& Holmes, 2013; Oksanen et al. , 2013; Bolyen et al. , 2018).

\section{Taxonomic diversity analysis}

Analysis of taxonomic diversity was done partially in QIIME2 and partially in R v.3.6.1 using the packages 'qiime2R', 'phyloseq', 'indicspecies', 'vegan', 'ape' and dependent packages. Network analysis of Bray-Curtis dissimilarity of community diversity between demographic groups was conducted to find groups of seals which 
were more or less similar as well as seal demographics that were more or less similar in their community to the salmon sampled as has been done in other microbiome studies (Ling et al. , 2016; Layeghifard et al. , 2017). Taxonomic differences between groups were determined using analysis of composition of microbiomes (ANCOM) using the 'composition' plugin in QIIME 2. The ANCOM analysis was used to find shared OTUs between seals sampled at the FF and the salmon in the farms (Mandal et al. 2015). An indicator species analysis (ISA) was used to find OTUs which were significantly associated with one group over others, based significant abundance differences between groups of seals and salmon microbiomes, and determine if any OTUs were observed in two or more groups more than in the others (De Cáceres et al., 2010; Mandalet al. , 2015). The ISA was conducted using the 'multiplatt' function in the $\mathrm{R}$ package 'indicspecies' to identify the taxa determined to be indicator species for the different groups of seals, specifically males that forage at salmon farms (De Cáceres et al. , 2010).

\section{Results}

\section{Sequence results}

For all seal samples $(\mathrm{n}=223)$ there were a total of 4,497,302 sequences, with the mean number of sequences in a sample being 20,167 (min: 3,925 - max: 165,000). After denoising, the mean number of paired-end sequences per sample was 3,836 (min: 514 - max: 24,703). After merging sequences, and removing chimeras and clustering to find representative sequences, the total number of representative sequences was 2,119 with a mean length of 471.1 and a range of 277 (min length: 300, max length: 577). For the dataset containing both seals and the salmon gut microbiota $(\mathrm{n}=526)$, there were a total of $64,501,265$ sequences, with the mean number of seqs per sample at 122,626 (min: 0, max: 423,448). After denoising, the mean number of pairedend sequences was 48,162.13 (min: 0, max: 247057). The mean number of merged sequences per sample was 46,236.76 (min: 0, max:243,669). After removing chimeras and post clustering, there were a total of 16,003 representative sequences, with a mean length of 453.35 and a range of 248 (min length: 300, max length: $548)$.

\section{Seal sample alpha, beta diversity, and network analysis}

The phylogenetic diversity determined by Faith phylogenetic diversity showed that community biodiversity was significantly different between multiple subgroups of seals. The phylogenetic biodiversity measured was significantly different in all pairwise comparisons between seals captured at fish farms, those in the region surrounding fish farms, and those sampled in Bass Strait (Figure 2a). Interestingly, the phylogenetic biodiversity, the Shannon diversity index, the Simpsons index, evenness, and number of observed OTUs were not significantly different between adults sampled and the juveniles sampled. However, significant diversity differences were seen from all diversity measures when comparing all demographic groups (control juveniles, control adults, in region adults, and males captured at farms), the Shannon diversity seen in each group are shown in figure $2 \mathrm{a}$ and the phylogenetic diversity are seen in figure $2 \mathrm{~b}$. As these demographic groups showed the most consistently significant alpha diversity differences between samples, they were used as the groups tested for taxonomic diversity analyses downstream.

The PERMANOVA of the UniFrac distances revealed significant community differences between multiple groups of seals. Significant differences between groups sampled: males sampled at fish farms, those sampled from the wild control group, and those sampled in the region around fish farms (overall: pseudo- $\mathrm{F}=10.734$, $\mathrm{p}=0.001$, distance between fish farm and control: pseudo- $\mathrm{F}=12.608, \mathrm{p}=0.001$ ). Seals sampled in different regions of Tasmania had significantly different community diversity (overall: pseudo- $\mathrm{F}=5.45, \mathrm{p}=0.001$ ), with the greatest differences seen between Bass Strait seals and those sampled in the south of Tasmania in the regions where salmon farms are located (pseudo- $\mathrm{F}=8.12, \mathrm{p}=0.001)$. Juveniles and adult seals had significantly different community diversity as well (pseudo- $\mathrm{F}=10.64, \mathrm{p}=0.001$ ). The unweighted Unifrac PCoA of the seal microbial communities showed the dissimilarity of communities between groups, with seals sampled at fish farms clustering somewhat removed from the rest of samples (supplemental figure 1). The weighted UniFrac distances show a community taxonomic differences between groups, with most males at fish farms forming an apparent cluster, as well as a cluster of juvenile seal microbiomes, along with overlap 
seen in all group of seals (figure 3 ).

Network analysis of the Bray-Curtis distances revealed that males at fish farms had two distinct groups of microbial communities, one of which, was more dissimilar to the rest of seal samples (figure 5). The network analysis identified strong relatedness among seals overall. However, the control seals seemed to cluster with the greatest similarity, while the males from fish farms, and the seals sampled in the region around farms, were more divergent from the rest of the seals samples.

\section{Taxonomic diversity differences within the seals in Tasmania}

Taxonomic differences between groups of seals sampled were analysed for common and significantly different OTUs found within and between seals sampled. As multiple subsets of adults were sampled, the relative abundance of bacterial phyla represented in the four groups of seals are shown in figure 4. As the amount of Proteobacteria are represented in different relative abundances in all groups, the relative abundance of families represented in the Proteobacteria in seal samples were calculated (supplemental figure 2). Overall, the males sampled at the farms and the animals sampled in the regions around farms had more diversity within the Proteobacteria phylum seen than the other groups of seals. Juveniles had relatively more Firmicutesthan all groups of adults, but the relative abundance of this phyla were similar among all adult seal groups.

The ANCOM results presented show the taxonomic assignments of OTUs represented in seal microbiomes with significant difference between groups, the number of sequences in $75 \%$ and $100 \%$ of the seals in that group, the F-statistic is a measure of effect size of the difference between groups, and the W-statistic which is a count of the sub-hypotheses passed during all of the pair-wise tests which make up the ANCOM (table 1). The ANCOM analysis of seals revealed 54 OTUs that were significantly different between males at salmon farms and adults sampled in the region, control adults and control juveniles. Of the 54 OTUs, 21 were OTUs that were significantly different between males at fish farms in higher frequency than the other groups of seals (table 1). Genera that were found to be more significantly associated with salmon farm frequenting seals were Campylobacter, Psychrobacter, andProteiniphilum; the rest are identified in table 1 . Three of the OTUs found significantly different between seals were also represented in the salmon samples, the assigned taxonomy to these three OTUs were Cetobacterium ceti (279 sequences in 7 salmon samples),Photobacterium damselae subsp. piscicida (4 sequences in 1 salmon sample), and Pelomonas sp. (1202 sequences in 73 salmon samples). The ANCOM analysis also identified an OTU assigned asPelomonas sp. that was found in higher frequency in males at fish farms and adults sampled in the region around fish farms than in other seals (table $1)$.

Three OTUs included in the ANCOM results were assigned to an unclassified genus (16S rRNA gene sequence 91\% similar to Ornithobacterium rhinotracheale (family Weeksellaceae, phylum Bacteroidetes ) which had multiple isolates cultured from the seals captured at fish farms during this study, these OTUs were found significantly more in the males from salmon farms, and were not found in any of the juvenile animal microbiomes during the ANCOM analysis (table 1). As classification of this unclassified Weeksellaceae genus is ongoing, it will be referred to from hereon as "unclassified Weeksellaceae genus" in this study. This novel taxon was assigned to 101 OTUs total, and 16724 sequences were found in all seals sampled, only 350 of those were in pups (2.0\%) present in low sequence numbers in 19 of the 50 pups samples (38\%), while the majority of the sequences were in males sampled from the salmon farms; 15,013 sequences (89.7\%) in 37 of the 56 males sampled ( $66 \%$ of males), then in the adults sampled in the region (1189 sequences, in $15 / 53$ in region adults $28 \%$ ) and in low numbers in the adults sampled as controls, only 164 sequences in 15 out of 60 seals.

\section{Seals compared to farmed salmon - alpha and beta diversity}

There were significant Faith phylogenetic biodiversity differences between the seals sampled and the salmon (Kruskal-Wallis pairwise $\mathrm{H}=55.609$, p-value $<8.85 \times 10^{-14}$ ) as well as significant differences between adult seals, juvenile seals and salmon (overall $\mathrm{H}=54.280$, p-value $=1.63 \times 10^{-12}$ ) with the largest differences seen between adult seals and salmon $\left(\mathrm{H}=41.77\right.$, $\mathrm{p}$-value $\left.<1.026 \times 10^{-10}\right)$ and juvenile seals and salmon $(\mathrm{H}=23.7$, p-value $<1.12 \times 10^{-6}$ ) (supplemental figure 3$)$. Community evenness was significantly different between seals 
and salmon seen by Pielou's evenness index showing greater evenness of samples in seals (Kruskal-Wallis $\mathrm{H}=2016.94$, p-value $\left.<6.38 \times 10^{-47}\right)$. The Shannon diversity index was higher overall in seals compared to salmon, showing an overall higher richness and evenness $\left(\mathrm{H}=134.1\right.$, $\mathrm{p}$-value $\left.<5.19 \times 10^{-31}\right)$.

The PERMANOVA results of the Unweighted UniFrac distances showed a significant difference in community diversity between seals and salmon (pseudo- $\mathrm{F}=135.179$, p-value $<0.001$ ). The PERMANOVA results comparing salmon to all groups of seals show significant differences between salmon and all groups (overall: pseudo- $\mathrm{F}=40.42, \mathrm{p}$-value $<0.001)$ the greatest distance was between the control adults and the salmon (pseudo- $\mathrm{F}=68.505, \mathrm{p}$-value $<0.001$ ) and then control juvenile seals to salmon (pseudo- $\mathrm{F}=46.691$, $\mathrm{p}$ value $<0.001)$. The males sampled at the salmon farms had the lowest difference in unweighted Unifrac distance to the salmon (pseudo- $\mathrm{F}=38.421$, $\mathrm{p}$-value $<0.001$ ). The principal coordinate analysis of the unweighted UniFrac distances revealed males sampled at the farms were qualitatively closer in microbial community diversity to the salmon samples than any of the other groups of seals (figure 6). The weighted UniFrac PERMANOVA showed that the quantitative community dissimilarity between seals and salmon were significantly different (pseudo-F=272.425, p-value $<0.001$ ). However, the greatest dissimilarity between salmon and a group of seals was that of the control adults and control juveniles sampled far away from fish farms (Adults: pseudo- $\mathrm{F}=161.84$, $\mathrm{p}$-value $<0.001$; Juveniles: pseudo- $\mathrm{F}=108.69$, $\mathrm{p}$-value $<0.001)$ while the seals with the least amount of dissimilarity to the salmon were the seals sampled at fish farms (pseudo- $\mathrm{F}=93.94$, $\mathrm{p}$-value $<0.001$ ).

Network analysis, using Bray-Curtis distances as metrics of dissimilarity revealed community differences between the demographics, highlighting the community dissimilarity between seals and salmon, but also, highlighting that the communities found in males sampled at FF were the least dissimilar to the salmon in the farms (figure 7). However, no connections were seen between salmon and seals. More connections were apparent between salmon samples than were witnessed in seals, showing that potentially salmon microbiomes are more static than those in seals. There were three juvenile animals which had higher similarity with the salmon than the rest of the control animals, however the males sampled at FF had many more samples with higher similarity to the salmon samples.

Taxonomic diversity results, ISA, ANCOM

The relative abundance of OTUs at the phylum level in salmon and the different seal demographic groups are shown in figure 8 . These results show that male seals foraging at fish farms have different abundant species compared to the other adults sampled; with an increase inBacteroidetes and Proteobacteria compared to all other seals sampled (figure 8). Salmon have microbiota that are dominated by Proteobacteria .

The ISA of the seals OTUs compared between groups, revealed 189 OTUs which were significantly different between groups (supplemental table 1). The ISA of all samples including salmon revealed 531 OTUs which were significantly different between all groups. The ISA comparing seals to animals at or in the region around farms (salmon and seals) found 143 OTUs which were significantly related to being from an animal at/near fish farms, and 223 OTUs which were significantly related to being found in animals in Bass strait. The OTUs found through the ISA and the taxonomic assignments of them showed vast differences, which are seen in the supplemental table 1 . The males caught at fish farms had more diversity amongst the taxa represented in their indicator species than any other group of seals, except for in the phylum Firmicutes which were more diversely represented as indicator species in juveniles and in the adults sampled in the regions around fish farms.

The ISA elucidated which representative taxa in Proteobacteriawere significantly different between the males sampled at the salmon farms compared to all other groups. These included OTUs which were classified as Psychrobacter, Otariodobacter, Suttonella, Xanthomonadaceae, and Burkholderiaceae. Additionally, adults sampled in the region around farms had OTUs, which were significantly related to this group, and were classified as Edwardsiella hoshinae and Plesiomonas shigelloides. Psychrobacter andSuttonella were found significantly related to males sampled at the farms in both ANCOM and ISA analyses. All but two of the OTUs found in the ANCOM analysis were included in the ISA, therefore showing agreement (52/54 OTUs agreed) and identified an additional 137 OTUs which were significantly different between groups of seals. 
Through comparing the ISA results to the taxonomic assignments, there were four OTUs which were significantly different between the control seals (adults and juveniles) and the animals at or near the salmon farms (salmon, males caught at a fish farm, and adults sampled in the region). These OTUs were also associated with both salmon and seals during the ISA when comparing all samples. Two of the four OTUs were associated with adults sampled in the region and were also found in salmon hind gut digesta samples, while the other two were associated with males at fish farms. The OTUs which were found in adults in the region and salmon, were Clostridium sensu stricto $1 \mathrm{sp}$. (Control vs. Fish farm animals: p-value $=0.013$, indicator value $=0.27$; In Region adults vs. all other groups of seals: $\mathrm{p}$-value $=0.001$, indicator value $=0.427)$ and Plesiomonas shigelloides (Control vs. Fish farm animals: p-value $=0.037$, indicator value $=0.228$; In Region adults vs. all other groups of seals: $\mathrm{p}$-value $=0.001$, indicator value $=0.452$ ). The two OTUs in the males sampled at fish farms included one OTU classified in the class Bacteroidia while the other OTU was classified as a member of the unclassified Weeksellaceae genus.

All OTUs which were classified as representing the unclassified Weeksellaceae genus were significantly related to males at fish farms compared to all other groups sampled, except one OTU which was significantly related to control juveniles, but represented by only 81 sequences in all seals (four pups, one male from a fish farm and one long-nosed fur seal which was necropsied). The ANCOM analysis also identified OTUs classified as unclassified Weeksellaceae genus were more significantly prevalent in the males at fish farms than in any other group. While the specific OTUs found through the ANCOM analysis of the seals only were not present in the salmon samples, there were 157 seqs of 4 OTUs in 7 salmon samples that were assigned to unclassified Weeksellaceae genus and were shared with 1 seal sampled in the region around salmon farms. The ANCOM analysis comparing the composition of the salmon microbial communities to the male seals captured at the fish farm revealed OTUs which were found in both and are shown in table 2 with the number of sequences represented in each group ( $100 \%$ of all seals and salmon). Many OTUs assigned to the same taxonomy were found through ANCOM, and the majority of OTUs found in both males caught at fish farms and salmon were classified in the phylumProteobacteria .

\section{Discussion}

This study is the first to document taxonomic community shifts and biodiversity changes in microbial communities in a wild pinniped gut in relation to marine salmon aquaculture activity in a shared habitat with human development. Overall, alpha diversity differences seen between regions, as well as between animals sampled at fish farms, and all other seals, suggests that diet and regional differences may impact overall biodiversity in Australian fur seal gut microbiomes more than age class does. However, beta diversity differences suggest that community makeup, rather than overall biodiversity, was implicated in the community diversity difference seen between demographic groups of seals. Pups and adults differed in their community makeup, which is supported by previous research indicating the community shifting as an animal ages (Smith et al. , 2013). Differences were seen between each group of seal sampled, however, the biggest taxonomic difference between seals were found between adults sampled at the control sites and the males sampled at fish farms.

Interestingly, this study shows that the microbial community of pups was more similar at the phylum relative abundance level to the males sampled at fish farms than the control adults sampled at the rookeries where the pups were also sampled and the seals sampled from haul-outs near salmon farming activities. Analysis of the genus level differences between all adult groups sampled and pups indicated that the lower taxonomic differences in the phyla present, was very different between adults and pups, this is supported by previously published work on fur seal pup microbiota in the region (Grosser et al. , 2019). This was especially apparent by the taxa represented in the phylumProteobacteria for the different groups of seals sampled. Pups at the time of sampling were between 1-2 months old and had never left the rookery island, nor swam beyond small pools at the rookery, therefore they would have encountered most enteric bacteria from their mothers, both from the milk, which is their only food source at that age, and through suckling after females have moved through the haul-out and picked up bacteria from the fecal remains found throughout the rookery. They are also likely to have limited exposure to environmental bacteria compared to the adult seals sampled, as adults 
have already migrated around Tasmania, thus encountering a wider variety of prey species and bacteria in the environment.

The major differences found among the seals sampled in Tasmania, were seen between regions and the seals foraging at fish farms compared to seals sampled in Bass strait. At this basic biodiversity level foraging on farm salmon/around farms does appear to shift microbiota and change the diversity and community of the seal microbiome. Both alpha and beta diversity differences among seals were most evident between seals sampled at the salmon farms and all other groups of seals sampled. While this study included samples across the Tasmanian habitat, age range and variable distances from salmon farms, it was limited to seals from the end of 2017 through the beginning of 2019. Therefore, changes overtime and sex specific differences were not captured. It is likely that male seals are over-represented compared to females in the haul-out samples in regions around farms, due to their natural behaviour to forage in more southerly regions, however, as they are the only sex which forage at the farms this limitation may have led to capturing of more fine-scale changes based on male diet and regionality rather than overall differences between sexes. Further work would need to include multiple years, representative samples from the rest of the species range, and targeted sampling of adult females and males in known proportions.

The salmon microbiota samples included in this study, were a representative sampling of all farmed salmon in Tasmania in net pens, as known from previous research on the entire salmon population, therefore the differences seen between the seals and salmon are likely representative of differences between these two populations overall in the region (Zarkasi et al. , 2014; 2016; Neuman et al. , 2016).While the alpha and beta diversity differences between salmon and seals overall were high, as expected, due to different internal conditions found between fish and mammals, it is interesting to note that the group of seals least dissimilar to the salmon at this level of analysis were the males sampled at the farms. (Zarkasi et al., 2014; 2016; Neuman et al. , 2016). This was supported by the taxonomic analysis which revealed which two most abundant phyla were over-represented in seals foraging at fish farms, compared to all other groups of seals. The increase in Proteobacteria andBacteroidetes, indicates that these bacteria may be more prevalent in the area around farmed salmon leases or are being transmitted to the seals via predation events on salmon. The later appears to be more likely as the adults sampled in the same region of the fish farms, but not directly at the farms themselves, had similar abundances of Bacteroidetes, and a slight increase ofProteobacteria compared to the control adults, however still both were less abundant than the males sampled at the farms.

Community differences were analysed further by implementing ISA. This revealed that male seals from fish farms appear to have bacterial taxa which are not typical in the rest of the fur seal population sampled during this study. An unclassified genus of bacteria designated unclassified Weeksellaceae genus, also successfully cultured, was found to be considerably more prevalent in the males sampled from fish farms. This taxon belongs to the family Weeksellaceae withinBacteroidetes, and clearly represents a novel group that potentially could have biological significance in seals but requires further study. Through the ANCOM analysis and the ISA it was revealed that the OTUs which were assigned to unclassified Weeksellaceaegenus isolate sequences, from the isolate database, were also found more prevalently in males from salmon farms than any other group of seal, and were only found in limited numbers in both adults in and outside of the regions around salmon farms as well as one OTU in four pup samples (supplemental table 1). The specific OTUs found through the ANCOM analysis of the seals alone, were not present in the salmon samples, and only one seal shared an OTU with the salmon sampled which classified as unclassified Weeksellaceae genus. So, while it is interesting that unclassified Weeksellaceae genus sequences appear over-represented in the males sampled at fish farms, it is unclear through this research, whether it is derived from the salmon or from the ecosystem they share. It is possible the bacteria are more readily able to colonize and grow in the fur seal gut, and thus, are not retained by salmon. It is unknown if they occur on the salmon skin or gills, as microbial community analysis on farmed salmon in this region is limited to the gastrointestinal tract.

Microbes that are conserved specifically between seals foraging at the farms and farmed salmon were seen in the ISA, and through the ANCOM analysis of the seals compared to the OTU table for the salmon samples; they were represented by $P$. shigelloides and members of the genusClostridium sensu stricto (cluster 1) which 
were significantly more frequently found in males sampled at the farms and those sampled in the regions around the farms, than the control seals sampled in Bass strait. These OTUs may be good indicators for the aquaculture input into the wild system, however, the frequency was low in both salmon and seals. This could be due to many factors related to the host-microbe interactions, the time each were sampled, or the relatively small abundance of these genera in the system overall, alternatively, they could be more commonly associated with the microbiota of the salmon skin or gills, which are yet unknown.

The taxonomic analysis revealed different OTUs found between the demographic groups of seals with varying levels of relative abundancies. One such OTU was identified as a representative of the Gram-negative genus Psychrobacter, which include species that are commonly found in saline environments, and grow at low temperatures (Bozalet al. , 2003; Bowman, 2006). As species of Psychrobacterhave been identified as opportunistic pathogens, and in some cases virulent pathogens, in humans and animals, including opportunistic infections in farmed salmonids, further identification of the these species in seals could lead to a better understanding of potential pathogens moving between animals and the environment at this interface (Hisar et al. , 2002; Romanenko et al. , 2004; Bowman, 2006). While the OTUs in this genus, found through the ISA, were significantly related to the microbiomes of male seals at the fish farms, other OTUs assigned to the Psychrobacter genus were found significantly more in salmon. Their significance as indicator species in males foraging on farmed salmon, more than any group of seals tested, as well as their presence in salmon, could show that these are temperature tolerant species and/or they are being shared at this interface. None of the OTUs which were assigned to the genus Psychrobacter had a species assignment, which may suggest there is a novel species being shared at this interface which has yet to be cultured and speciated.

The ANCOM analysis and ISA, which included the salmon microbiomes, found OTUs which were shared between seals and salmon. The culmination of this analysis revealed OTUs which were found significantly more in seals sampled at or near the farms and ones in those groups which were shared between them and salmon, these OTUs were mostly representatives ofFirmicutes and Proteobacteria. Among these OTUs, the fish pathogen Photobacterium damselae subsp. piscicida was identified, as well as a potential pathogen for mammals Klebsiella pneumoniae, along with P.shigelloides, and Clostridiumassigned OTUs were also observed (Romalde, 2002; Struve \& Krogfelt, 2004). All of these taxa were represented by other OTUs found in many seal microbiomes, and are commonly found in the marine environment, and thus this presence in salmon could be due to exposure through seals defecating in the area around farms, and/or found in the environment semi-regularly, thus exposing both species regardless of direct interaction. It is unknown from this study if they originate in the environment, the salmon or the seal guts, thus further characterisation of these bacteria from both would need to be done to determine directionality.

While $K$. pneumoniae is a typical enteric species in seals, virulent strains have caused disease in seals and humans, and the frequency of $P$. damselae in seals is largely unknown, however, is found in the marine system quite frequently, but this bacterium is known to cause infections in a wide array of marine animals including cases of incidental infection in cetaceans (Vedros et al. , 1982; Bucket al. , 1991; Castinel et al ., 2007; Rivas et al. , 2013; Di Francesco et al. , 2016). These species may be of concern for the health of wild and aquaculture fish populations, potentially marine mammals and humans that interact with them, as all are at risk of incidental infection of virulent strains of both taxa in other regions where these bacterium are found, however, little evidence was obtained during this study to ascertain the level of risk (Dryden et al. , 1989; Fouz, 1992; Tang \& Wong, 1999; Austin \& Austin, 2012).

The potential for pathogen transmission to and from aquaculture salmon and human development should be studied further. This study was limited to only one wild population of fur seals which are known to forage regularly on farmed salmon stocks, while other seabird, marine mammal, shark and wild fish species also interact with farmed salmon. The full extent of risk to wildlife is not known from this study, and the risk to salmon exposed to regular wild seal predation and run off from human development has been limited to bacterial shedding from seal defecation in the same area during this study. Through this study it is suggested that foraging on farmed salmon, and in the region around fish farms, affects the diversity and taxonomic make-up of microbial communities in seals. Bacterial sharing between salmon and seals has been documented 
through this research, and further monitoring of potential pathogens in this system should be undertaken at this interface in Tasmania and at similar wild predator and aquaculture interfaces globally.

\section{Author Contribution}

Erin D'Agnese - primary researcher, conducted all lab work, $80 \%$ collections, performed majority of analysis of microbiome data, and wrote the bulk of the manuscript.

Ryan McLaughlin - Assisted with data analysis of microbiome data and performed some of the more complex/large computational analyses. Helped in making the isolate sequence database, and throughout data analysis.

Mary-Anne Lea - Chief Investigator on the collaborative project which allowed for the sampling of live male seals at the farms. Involvement in idea development and manuscript prep.

Esteban Soto - Involved in editing/revising the manuscript and intellectual involvement in microbial interpretation

Woutrina Smith - Co-supervisor of the project. Provided financial support of project, involved in idea development, and manuscript prep.

John Bowman - The lead supervisor with the most experience in the field on the project, provided the salmon data required to complete project. Provided financial support for the project. Project was undertaken in his lab, under his supervision.

\section{Funding}

Funding for this project was provided by the University of Tasmania, Tasmania Institute of Agriculture, University of California, Davis, Laboratory of Woutrina Smith, the Australian Department of Education for Endeavour fellowship funding, and the University of Tasmania, College of Science and Engineering grant funding.

\section{Acknowledgments}

We would like to thank The Marine Conservation Program and Princess Melikoff Trust Marine Mammal Conservation Program within the Department of Primary Industries, Parks, Water and Environment, for sampling support for the fur seals around Tasmania, specifically Sam Thalmann, Annie Phillips and Cloe Cummings. We thank staff at Tassal Pty Ltd for their assistance in sampling the adult male seals.

\section{References}

Ahmed, W. (2007). Limitations of library-dependent microbial source tracking methods. Journal of the Australian Water Association FEBRUARY, 40.

Alfano, N., Courtiol, A., Vielgrader, H., Timms, P., Roca, A. L., \& Greenwood, A. D. (2015). Variation in koala microbiomes within and between individuals: effect of body region and captivity status.Sci Rep, 5 , 10189 .

Allen, H. K., Bayles, D. O., Looft, T., Trachsel, J., Bass, B. E., Alt, D. P., . . Casey, T. A. (2016). Pipeline for amplifying and analyzing amplicons of the V1-V3 region of the $16 \mathrm{~S}$ rRNA gene. BMC research notes, 9 (1), 380 .

Austin, B., \& Austin, D. A. (2012). Bacterial fish pathogens : Springer.

Barko, P. C., McMichael, M. A., Swanson, K. S., \& Williams, D. A. (2018). The Gastrointestinal Microbiome: A Review. J Vet Intern Med, 32 (1), 9-25.

Bolyen, E., Rideout, J. R., Dillon, M. R., Bokulich, N. A., Abnet, C., Al-Ghalith, G. A., . . Asnicar, F. (2018). QIIME 2: Reproducible, interactive, scalable, and extensible microbiome data science (2167-9843). Retrieved from 
Bourne, D. G., Dennis, P. G., Uthicke, S., Soo, R. M., Tyson, G. W., \& Webster, N. (2013). Coral reef invertebrate microbiomes correlate with the presence of photosymbionts. The ISME journal, 7 (7), 1452.

Bokulich, N. A., Kaehler, B. D., Rideout, J. R., Dillon, M., Bolyen, E., Knight, R., .. \& Caporaso, J. G. (2018). Optimizing taxonomic classification of marker-gene amplicon sequences with QIIME 2's q2-featureclassifier plugin. Microbiome, 6(1), 90.

Bowen, W., \& Lidgard, D. (2013). Marine mammal culling programs: review of effects on predator and prey populations. Mammal Review, 43 (3), 207-220.

Bowman, J. P. (2006). The genus psychrobacter. The Prokaryotes: Volume 6: Proteobacteria: Gamma Subclass , 920-930.

Bozal, N., Montes, M. J., Tudela, E., \& Guinea, J. (2003). Characterization of several Psychrobacter strains isolated from Antarctic environments and description of Psychrobacter luti sp. nov. and Psychrobacter fozii sp. nov. International journal of systematic and evolutionary microbiology, 53 (4), 1093-1100.

Buck, J., Overstrom, N., Patton, G., Anderson, H., \& Gorzelany, J. (1991). Bacteria associated with stranded cetaceans from the northeast USA and southwest Florida Gulf coasts. Diseases of Aquatic Organisms, 10 (2), 147-152.

Callahan, B. J., McMurdie, P. J., Rosen, M. J., Han, A. W., Johnson, A. J. A., \& Holmes, S. P. (2016). DADA2: high-resolution sample inference from Illumina amplicon data. Nature methods, 13 (7), 581.

Castinel, A., Grinberg, A., Pattison, R., Duignan, P., Pomroy, B., Rogers, L., \& Wilkinson, I. (2007). Characterization ofKlebsiella pneumoniae isolates from New Zealand sea lion (Phocarctos hookeri ) pups during and after the epidemics on Enderby Island, Auckland Islands. Veterinary microbiology, 122 (1), 178-184.

Christian, N., Whitaker, B. K., \& Clay, K. (2015). Microbiomes: unifying animal and plant systems through the lens of community ecology theory. Frontiers in Microbiology, 6, 869.

De Cáceres, M., Legendre, P., \& Moretti, M. (2010). Improving indicator species analysis by combining groups of sites. Oikos, 119 (10), 1674-1684.

Di Francesco, G., Cammà, C., Curini, V., Mazzariol, S., Proietto, U., Di Francesco, C. E., . . Di Guardo, G. (2016). Coinfection by Ureaplasma spp., Photobacterium damselae and anActinomyces -like microorganism in a bottlenose dolphin (Tursiops truncatus ) with pleuropneumonia stranded along the Adriatic coast of Italy. Research in Veterinary Science, 105, 111-114.

Dryden, M., Legarde, M., Gottlieb, T., Brady, L., \& Ghosh, H. (1989).Vibrio damsela wound infections in Australia. Medical Journal of Australia, 151 (9), 540-541.

Fouz, B. (1992). Characterization of $V$. damsela strains isolated from turbot Scophthalmus maximus in Spain. Dis. Aquat. Org., 12, 155-166.

Geldreich, E. E., \& Litsky, W. (1976). Fecal coliform and fecal streptococcus density relationships in waste discharges and receiving waters. C R C Critical Reviews in Environmental Control, 6 (4), 349-369.

Grice, E. A., \& Segre, J. A. (2011). The skin microbiome. Nature Reviews Microbiology, 9 (4), 244.

Griffith, J. F., Weisberg, S. B., \& McGee, C. D. (2003). Evaluation of microbial source tracking methods using mixed fecal sources in aqueous test samples. Journal of Water and Health, 1 (4), 141-151.

Grosser, S., Sauer, J., Paijmans, A. J., Caspers, B. A., Forcada, J., Wolf, J. B. W., \& Hoffman, J. I. (2019). Fur seal microbiota are shaped by the social and physical environment, show mother-offspring similarities and are associated with host genetic quality. Mol Ecol, 28 (9), 2406-2422.

Gulland, J. A. (1987). Seals and fisheries: A case for predator control? Trends Ecol Evol, 2 (4), 102-104. 
Hall, M., \& Beiko, R. G. (2018). 16S rRNA gene analysis with QIIME2. InMicrobiome Analysis (pp. 113-129): Springer.

Hills, R. D., Jr., Pontefract, B. A., Mishcon, H. R., Black, C. A., Sutton, S. C., \& Theberge, C. R. (2019). Gut Microbiome: Profound Implications for Diet and Disease. Nutrients, 11 (7).

Hisar, O., Yanik, T., \& Hisar, S. A. (2002). Clinical And Pathological Investigation Of Psychrobacter Immobilis Infection In Rainbow Trour (Oncorhynchus Mykiss, Walbaum ).

Holyoak, M., Leibold, M. A., Mouquet, N., Holt, R. D., \& Hoopes, M. (2005). A framework for large scale community ecology.Metacommunities: spatial dynamics and ecological communities. The University of Chicago Press, Chicago , 1-31.

Huse, S. M., Ye, Y., Zhou, Y., \& Fodor, A. A. (2012). A core human microbiome as viewed through 16S rRNA sequence clusters. PLoS One, 7 (6), e34242.

Kembel, S. W., Jones, E., Kline, J., Northcutt, D., Stenson, J., Womack, A. M., . . Green, J. L. (2012). Architectural design influences the diversity and structure of the built environment microbiome. The ISME journal, 6 (8), 1469.

Kemper, C. M., Pemberton, D., Cawthorn, M., Heinrich, S., Mann, J., Wursig, B., . . Gales, R. (2003). Aquaculture and marine mammals: co-existence or conflict. Marine mammals: fisheries, tourism and management issues , 208-225.

Kirkwood, R., Pemberton, D., Gales, R., Hoskins, A. J., Mitchell, T., Shaughnessy, P. D., \& Arnould, J. P. (2010). Continued population recovery by Australian fur seals. Marine and freshwater research, 61 (6), 695-701.

Knights, D., Kuczynski, J., Charlson, E. S., Zaneveld, J., Mozer, M. C., Collman, R. G., . . Kelley, S. T. (2011). Bayesian community-wide culture-independent microbial source tracking. Nature methods, 8 (9), 761.

Layeghifard, M., Hwang, D. M., \& Guttman, D. S. (2017). Disentangling Interactions in the Microbiome: A Network Perspective. Trends in Microbiology, 25 (3), 217-228.

Ley, R. E., Lozupone, C. A., Hamady, M., Knight, R., \& Gordon, J. I. (2008). Worlds within worlds: evolution of the vertebrate gut microbiota. Nat Rev Microbiol, 6 (10), 776-788.

Ling, N., Zhu, C., Xue, C., Chen, H., Duan, Y., Peng, C., . . Shen, Q. (2016). Insight into how organic amendments can shape the soil microbiome in long-term field experiments as revealed by network analysis. Soil Biology and Biochemistry, 99 , 137-149.

Mandal, S., Van Treuren, W., White, R. A., Eggesbo, M., Knight, R., \& Peddada, S. D. (2015). Analysis of composition of microbiomes: a novel method for studying microbial composition. Microb Ecol Health Dis, 26,27663 .

Martinez-Guryn, K., Leone, V., \& Chang, E. B. (2019). Regional Diversity of the Gastrointestinal Microbiome. Cell Host \& Microbe, 26 (3), 314-324.

McMurdie, P. J., \& Holmes, S. (2013). phyloseq: an R package for reproducible interactive analysis and graphics of microbiome census data. PLoS One, 8 (4), e61217.

Miller, E. T., Svanbäck, R., \& Bohannan, B. J. (2018). Microbiomes as metacommunities: understanding host-associated microbes through metacommunity ecology. Trends in ecology $\&$ evolution, 33 (12), 926-935.

Mobsby, D. (2018). Australian fisheries and aquaculture statistics 2017 . Retrieved from Canberra, ACT:

Msangi, S., \& Batka, M. (2015). The rise of aquaculture: The role of fish in global food security. IFPRI book chapters, 61-72. 
Nash, C. E., Iwamoto, R. N., \& Mahnken, C. V. (2000). Aquaculture risk management and marine mammal interactions in the Pacific Northwest.Aquaculture, 183 (3-4), 307-323.

Neuman, C., Hatje, E., Zarkasi, K. Z., Smullen, R., Bowman, J. P., \& Katouli, M. (2016). The effect of diet and environmental temperature on the faecal microbiota of farmed Tasmanian Atlantic Salmon (Salmo salar L. ). Aquaculture Research, 47 (2), 660-672.

Oksanen, J., Blanchet, F. G., Kindt, R., Legendre, P., Minchin, P. R., O'hara, R., . . Wagner, H. (2013). Package 'vegan'. Community ecology package, version, 2 (9), 1-295.

Pacheco-Sandoval, A., Schramm, Y., Heckel, G., Brassea-Perez, E., Martinez-Porchas, M., \& Lago-Leston, A. (2019). The Pacific harbor seal gut microbiota in Mexico: Its relationship with diet and functional inferences. PLoS One, 14 (8), e0221770.

Patterson, H., Larcombe, J., Nicol, S., \& Curtotti, R. (2018).Fishery status reports 2018 . Retrieved from Canberra, Australia:

Pemberton, D., \& Shaughnessy, P. D. (1993). Interaction between seals and marine fish-farms in Tasmania, and management of the problem.Aquatic Conservation: marine and freshwater ecosystems, 3 (2), 149-158.

Rivas, A. J., Lemos, M. L., \& Osorio, C. R. (2013).Photobacterium damselae subsp. damselae, a bacterium pathogenic for marine animals and humans. Frontiers in Microbiology, 4, 283.

Robinson, S., Terauds, A., Gales, R., \& Greenwood, M. (2008). Mitigating fur seal interactions: relocation from Tasmanian aquaculture farms. Aquatic Conservation: marine and freshwater ecosystems, 18 (7), 11801188.

Rognes, T., Flouri, T., Nichols, B., Quince, C., \& Mahe, F. (2016). VSEARCH: a versatile open source tool for metagenomics. PeerJ, 4, e2584.

Romalde, J. L. (2002). Photobacterium damselae subsp. piscicida: an integrated view of a bacterial fish pathogen. International Microbiology, 5 (1), 3-9.

Romanenko, L. A., Lysenko, A. M., Rohde, M., Mikhailov, V. V., \& Stackebrandt, E. (2004). Psychrobacter maritimus sp. nov. andPsychrobacter arenosus sp. nov., isolated from coastal sea ice and sediments of the Sea of Japan. International journal of systematic and evolutionary microbiology, 54 (5), 1741-1745.

Scott, T. M., Rose, J. B., Jenkins, T. M., Farrah, S. R., \& Lukasik, J. (2002). Microbial source tracking: Current methodology and future directions. Applied and Environmental Microbiology, 68 (12), 5796-5803.

Smith, S. C., Chalker, A., Dewar, M. L., \& Arnould, J. P. (2013). Age-related differences revealed in Australian fur seal (Arctocephalus pusillus doriferus) gut microbiota. FEMS Microbiol Ecol, 86 (2), 246255.

Stevens, J. R., Newton, R. W., Tlusty, M., \& Little, D. C. (2018). The rise of aquaculture by-products: Increasing food production, value, and sustainability through strategic utilisation. Marine Policy, 90 , $115-124$.

Struve, C., \& Krogfelt, K. A. (2004). Pathogenic potential of environmental Klebsiella pneumoniae isolates. Environmental Microbiology, 6 (6), 584-590.

Tang, W., \& Wong, J. (1999). Necrotizing fasciitis caused by Vibrio damsela . Orthopedics, 22 (4), 443-444.

Unno, T., Di, D. Y., Jang, J., Suh, Y. S., Sadowsky, M. J., \& Hur, H.-G. (2011). Integrated online system for a pyrosequencing-based microbial source tracking method that targets Bacteroidetes 16S rDNA.Environmental Science 83 Technology, 46 (1), 93-98.

Unno, T., Jang, J., Han, D., Kim, J. H., Sadowsky, M. J., Kim, O.-S., . . . Hur, H.-G. (2010). Use of barcoded pyrosequencing and shared OTUs to determine sources of fecal bacteria in watersheds. Environmental Science 83 Technology, 44 (20), 7777-7782. 
Vedros, N. A., Quinlivan, J., \& Cranford, R. (1982). Bacterial and fungal flora of wild northern fur seals (Callorhinus ursinus ). Journal of Wildlife Diseases, 18 (4), 447-456.

Wiirsig, B., \& Gailey, G. (2002). Marine mammals and aquaculture: conflicts and potential resolutions. Responsible Marine Aquaculture. CAP International Press, New York, 45-59.

Xu, Z., \& Knight, R. (2015). Dietary effects on human gut microbiome diversity. The British Journal of Nutrition, 113 (Suppl 0), S1-S5.

Zarkasi, K. Z., Abell, G. C., Taylor, R. S., Neuman, C., Hatje, E., Tamplin, M. L., . . . Bowman, J. P. (2014). Pyrosequencing-based characterization of gastrointestinal bacteria of Atlantic salmon (Salmo salar L. ) within a commercial mariculture system. J Appl Microbiol, 117 (1), 18-27.

Zarkasi, K. Z., Taylor, R. S., Abell, G. C. J., Tamplin, M. L., Glencross, B. D., \& Bowman, J. P. (2016). Atlantic Salmon (Salmo salar L. ) Gastrointestinal Microbial Community Dynamics in Relation to Digesta Properties and Diet. Microbial Ecology, 71 (3), 589-603.

\section{Data Accessibility}

The DNA sequences for the seal microbiomes along with the metadata file used will be accessible via Dryad. The fastq files for the salmon DNA sequence data used are stored on NCBI BioProject Accession number PRJNA434528 and sequence read archive study number SRP133157.

Table 1. ANCOM results showing the taxonomic assignments OTUs that were found in high frequency among Males at fish farms and were significantly different between Males at fish farms and the other groups of seals. The F-statistic and W-statistics are shown for the differences among seal groups and the number of sequences in both $75 \%$ and $100 \%$ of seal samples in that group.

\begin{tabular}{llll}
\hline OTU taxonomic assignment & F-statistic & W-statistic & Males at \\
Epsilonbacteraeota; Campylobacter; uncultured bacterium & 33.54 & 2117 & 245.25 \\
Proteobacteria; Psychrobacter & 36.08 & 2117 & 158.25 \\
Fusobacteria; Fusobacterium & 35.02 & 2118 & 129.25 \\
Fusobacteria; Fusobacterium gut metagenome & 12.95 & 2111 & 101 \\
Bacteroidetes; "Pinnipedicolabacterium" & 31.49 & 2113 & 59.75 \\
Bacteroidetes; "Pinnipedicolabacterium" & 23.47 & 2113 & 51.75 \\
Bacteroidetes; Paludibacteraceae; H1; uncultured bacterium & 28.48 & 2113 & 46.5 \\
Proteobacteria; Neisseria & 24.24 & 2118 & 40.75 \\
Fusobacteria; Fusobacterium gonidiaformans & 24.81 & 2111 & 25 \\
Epsilonbacteraeota; Helicobacter & 9.95 & 2034 & 23 \\
Bacteroidetes; Alloprevotella & 9.02 & 2000 & 20.5 \\
Firmicutes; Ruminococcaceae; [Eubacterium] coprostanoligenes group gut metagenome & 20.27 & 2117 & 15.25 \\
Firmicutes; Lachnospiraceae; [Eubacterium] fissicatena group; uncultured bacterium & 39.81 & 2117 & 14.5 \\
Firmicutes; Erysipelotrichaceae; [Clostridium] innocuum group; uncultured bacterium & 11.33 & 2063 & 13.5 \\
Firmicutes; Clostridia; Clostridiales; Family XI; Ezakiella & 13.12 & 2076 & 10.5 \\
Firmicutes & 72.47 & 2118 & 9 \\
Proteobacteria; Suttonella & 17.8 & 2088 & 4.25 \\
Bacteroidetes; unclassified Weeksellaceae genus & 11.05 & 2011 & 0 \\
Bacteroidetes; Proteiniphilum; uncultured bacterium & 13.63 & 2078 & 0 \\
Proteobacteria; Escherichia-Shigella & 15.28 & 2089 & 0 \\
\hline
\end{tabular}

Table 2. The OTUs which were found through the ANCOM analysis comparing the microbial communities of male seals sampled from fish farms and the salmon microbial communities. All OTUs include are ones that were not found significantly different between salmon and seals captured at fish farms. Taxonomic 
assignments are reduced to the Phylum; Lowest taxonomic level assigned

\begin{tabular}{llll}
\hline OTU Taxonomic assignment & Salmon $100 \%$ & Seal $100 \%$ & W-statistic \\
Actinobacteria; Corynebacterium 1 & 69 & 22 & 132 \\
Actinobacteria; Cutibacterium & 1662 & 39 & 66 \\
Firmicutes & 78 & 71 & 80 \\
Firmicutes & 53 & 68 & 84 \\
Firmicutes; Bacillales & 1404 & 1696 & 69 \\
Firmicutes; Staphylococcus & 37 & 5 & 151 \\
Firmicutes; Enterococcus & 11 & 299 & 119 \\
Firmicutes; Lactobacillus iners AB-1 & 18 & 7 & 157 \\
Firmicutes; Lactococcus & 211 & 6 & 119 \\
Firmicutes; Lactococcus & 225 & 41 & 121 \\
Firmicutes; Clostridium sensu stricto 1 & 73 & 146 & 91 \\
Firmicutes; Clostridium sensu stricto 1 & 42 & 83 & 96 \\
Firmicutes; Clostridium sensu stricto 1 & 20 & 20 & 119 \\
Firmicutes; Clostridium sensu stricto 1 & 7 & 15 & 148 \\
Firmicutes; Erysipelatoclostridium ramosum & 42 & 38 & 110 \\
Fusobacteria; Cetobacterium ceti & 114 & 97 & 88 \\
Proteobacteria & 24 & 53 & 130 \\
Proteobacteria; Afipia & 35 & 68 & 103 \\
Proteobacteria; Rickettsiales & 7 & 455 & 112 \\
Proteobacteria; Aeromonas & 557 & 20 & 122 \\
Proteobacteria; Acidovorax & 41 & 116 & 120 \\
Proteobacteria; Burkholderiaceae & 14 & 44 & 107 \\
Proteobacteria; Pelomonas & 103 & 638 & 68 \\
Proteobacteria; Sutterella & 3 & 45 & 136 \\
Proteobacteria; Escherichia-Shigella & 59 & 44 & 83 \\
Proteobacteria; Escherichia-Shigella & 20 & 33 & 117 \\
Proteobacteria; Escherichia-Shigella & 37 & 11 & 124 \\
Proteobacteria; Klebsiella pneumoniae BIDMC 36 & 13 & 7 & 159 \\
Proteobacteria; Plesiomonas shigelloides & 108 & 41 & 97 \\
Proteobacteria; Acinetobacter & 22 & 196 & 120 \\
Proteobacteria; Photobacterium damselae subsp. piscicida & 5 & 7 & 137 \\
\hline
\end{tabular}




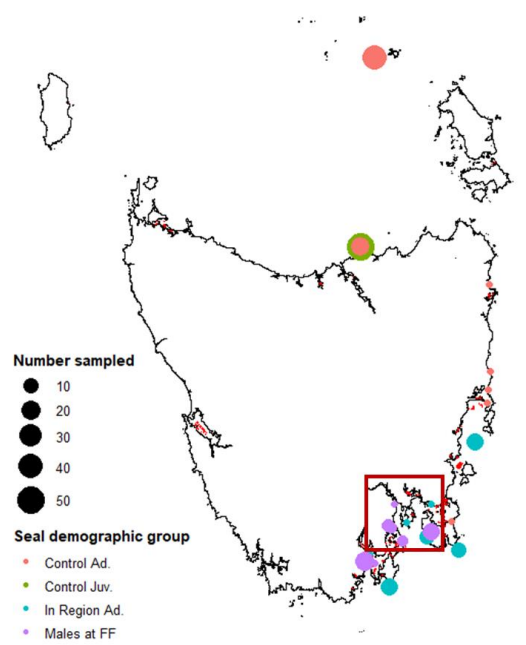

Figure 1. Map of sampling sites of fur seals around Tasmania, with colors representing demographic sampled and size of circle representing the number of animals sampled in that location. Red spots show aquaculture leases of all types, including Atlantic salmon, other fin fish (FF), and bivalves. The red square denotes the region including the major urban region of Hobart, Tasmania. 

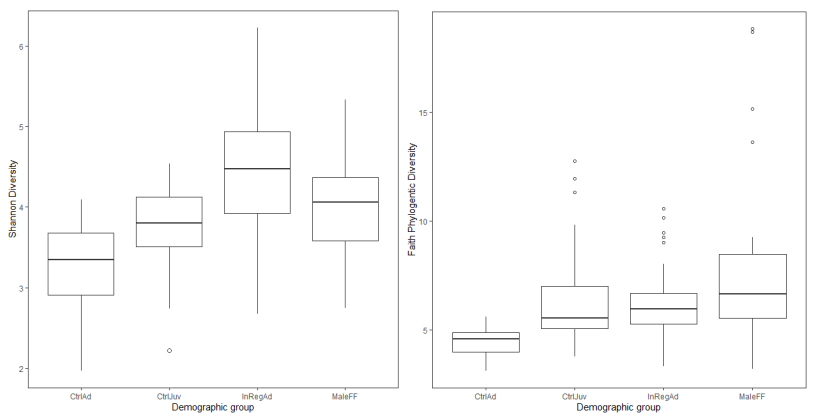

Figure 2. (2a) The Shannon diversity indices and (2b) the Faith Phylogenetic diversity indices found in each group of seals tested. males at fish farms (MaleFF), adults/scats sampled in the region directly around salmon (InRegAd), juveniles sampled outside of the regions around farms (CtrlJuv), adults/scats sampled outside the regions around farms (CtrlAd). 


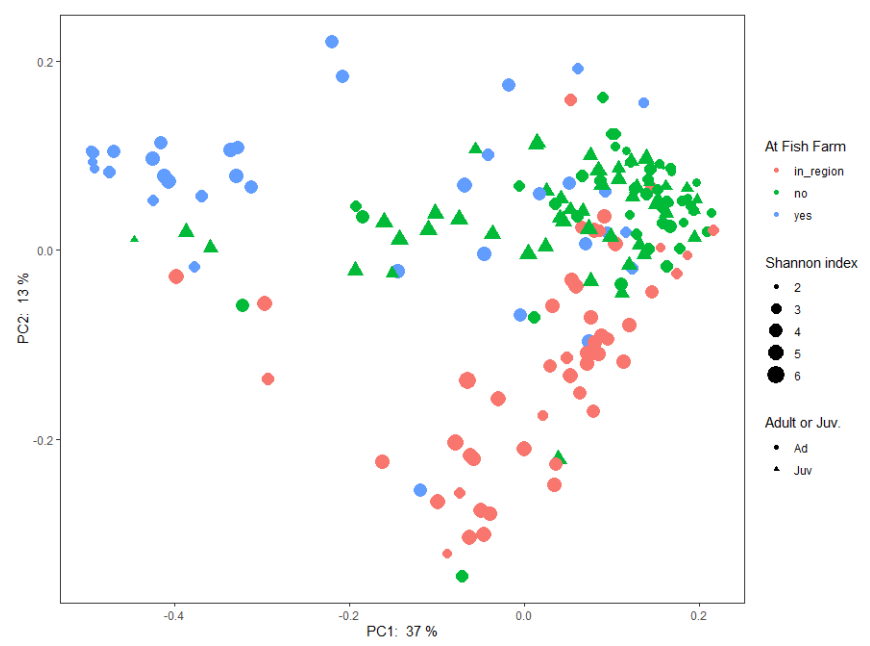

Figure 3. Weighted UniFrac distances of fur seals sampled in Tasmania including those sampled at fish farms, seals sampled in the regions around fish farms, and those sampled at the remote sites in Bass strait, differentiating between adults and juveniles fur seals sampled. The size of the samples reflects their Shannon diversity index. 


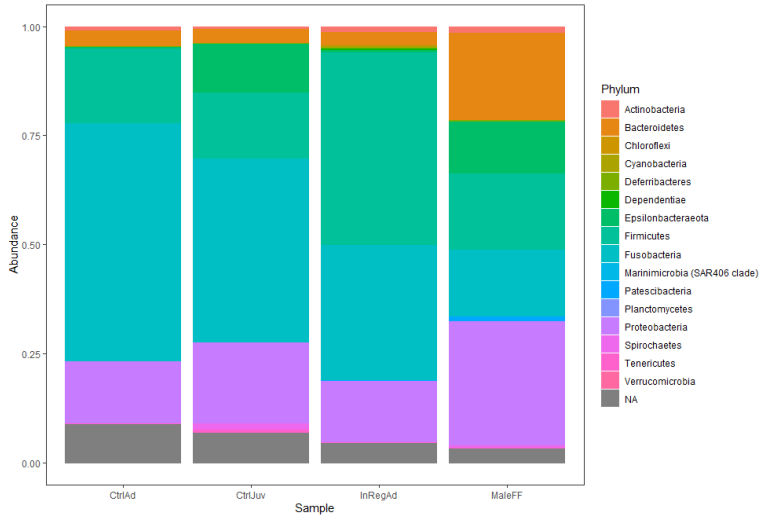

Figure 4. The relative abundance of bacterial phyla represented in the microbiomes of all four groups of fur seals sampled in Tasmania: males at fish farms (MaleFF), adults/scats sampled in the region directly around salmon (InRegAd), juveniles sampled outside of the regions around farms (CtrlJuv), adults/scats sampled outside the regions around farms (CtrlAd), NA refers to other/unidentified phylum. 


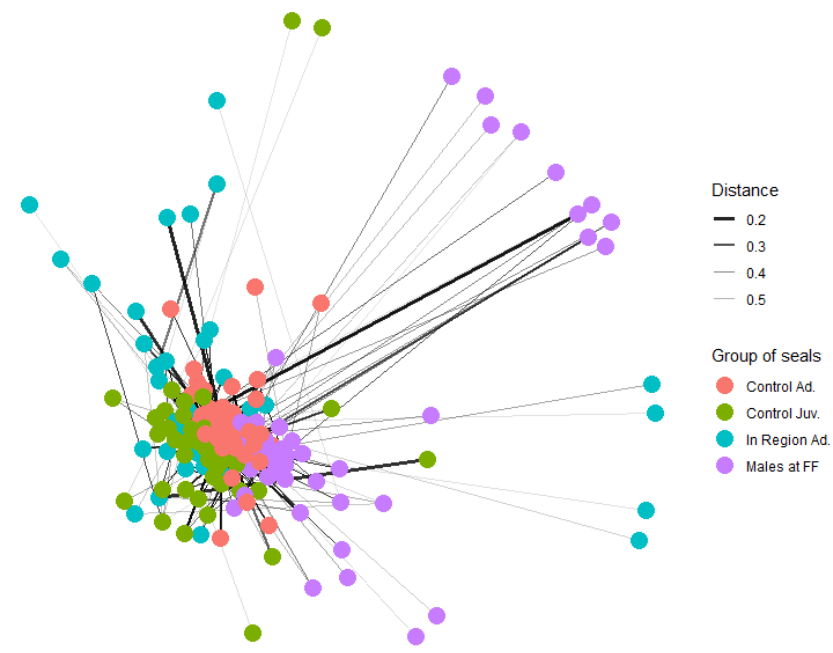

Figure 5. Network analysis of the Bray-Curtis dissimilarity distances of all seals sampled showing the samples with microbial communities that have different similarities among seals in Tasmania based on demographics: males at fish farms (MaleFF), adults/scats sampled in the region directly around salmon (InRegAd), juveniles sampled outside of the regions around farms (CtrlJuv), adults/scats sampled outside the regions around farms (CtrlAd). 


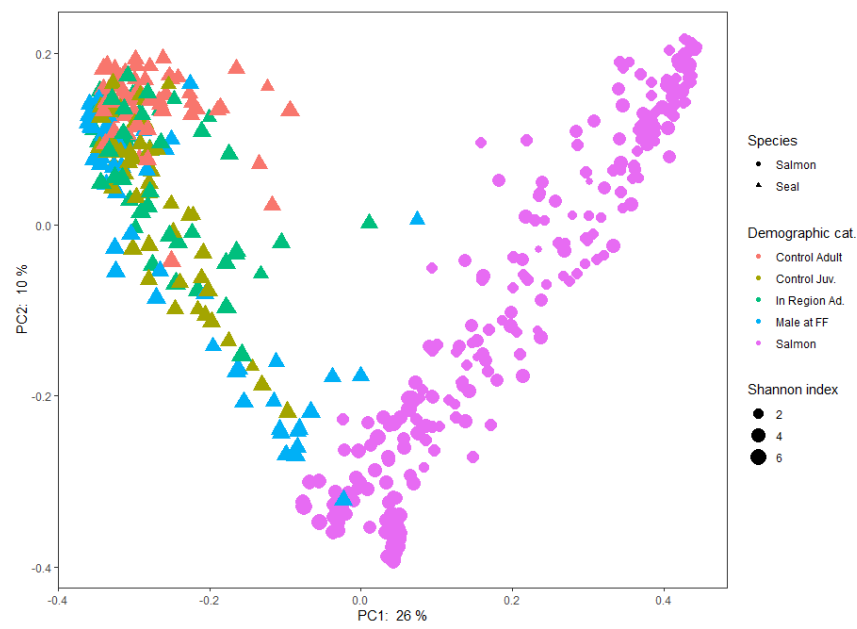

Figure 6. The unweighted UniFrac plot of the principal coordinates which explained the greatest differences in beta-diversity. Circles represent salmon, triangles represent seals. Blue denote being from a fish farm, green denotes being in the same region as fish farms and red denotes adults sampled not in the same region as fish farms, and yellow denote juvenile animals sampled outside the region around fish farms. 


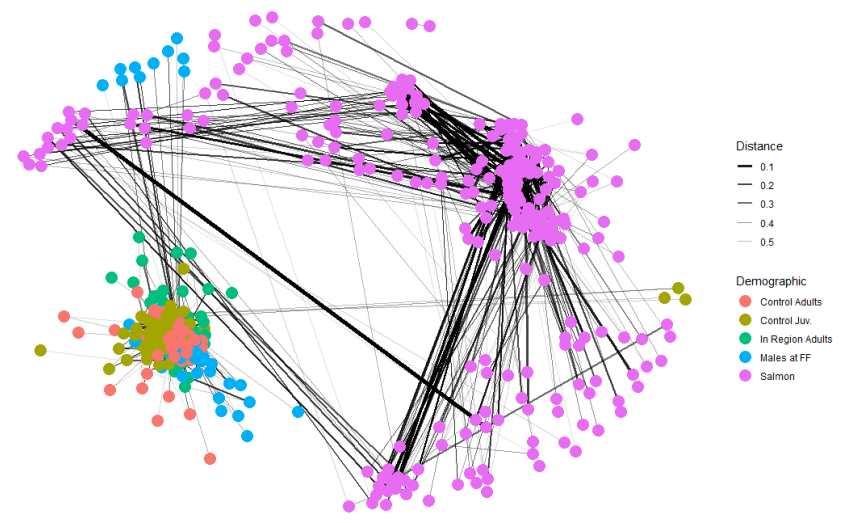

Figure 7. Network analysis of the community dissimilarity between all seal and salmon microbiomes sampled in Tasmania, using Bray-Curtis dissimilarity distances to calculate edges and a max-distance allowed of 0.6. Groups of seals represent males at fish farms (MaleFF), adults/scats sampled in the region directly around salmon (InRegAd), juveniles sampled outside of the regions around farms (CtrlJuv), adults/scats sampled outside the regions around farms (CtrlAd). 


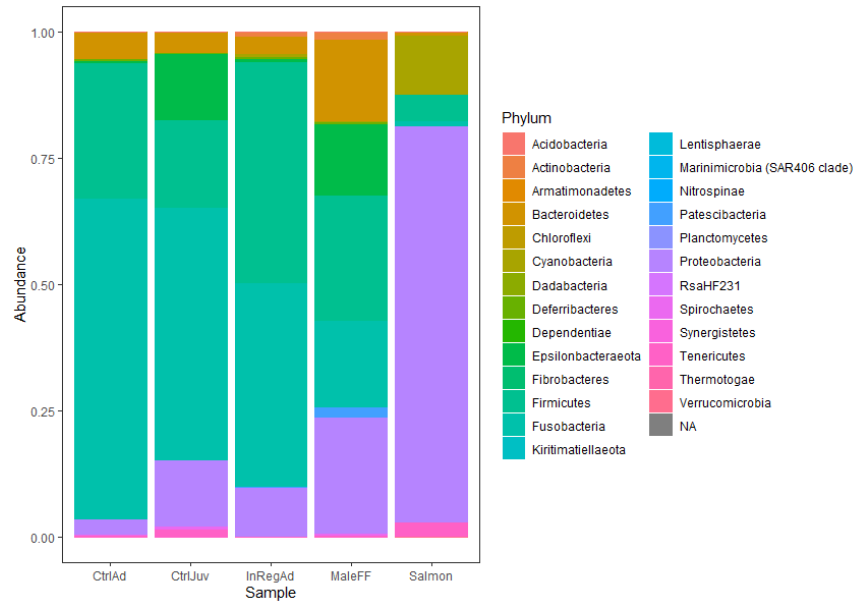

Figure 8. The relative abundance of the Phyla represented in seals and farmed salmon in Tasmania for each group of seals sample; males at fish farms (MaleFF), adults/scats sampled in the region directly around salmon (InRegAd), juveniles sampled outside of the regions around farms (CtrlJuv), adults/scats sampled outside the regions around farms (CtrlAd). 